This article has been accepted for publication in Monthly Notices of the Royal Astronomical Society (C: 2017 The Authors Published by Oxford University Press on behalf of the Royal Astronomical Society. All rights reserved. 


\title{
LOFAR discovery of an ultra-steep radio halo and giant head-tail radio galaxy in Abell 1132
}

\author{
A. Wilber, ${ }^{1 \star}$ M. Brüggen, ${ }^{1 \star}$ A. Bonafede,,${ }^{1,2}$ F. Savini,${ }^{1}$ T. Shimwell, ${ }^{3}$ \\ R. J. van Weeren, ${ }^{4}$ D. Rafferty, ${ }^{1}$ A. P. Mechev,${ }^{3}$ H. Intema, ${ }^{3}$ F. Andrade-Santos, ${ }^{4}$ \\ A. O. Clarke, ${ }^{5}$ E. K. Mahony, ${ }^{6,7}$ R. Morganti, ${ }^{8,9}$ I. Prandoni, ${ }^{2}$ G. Brunetti, ${ }^{2}$ \\ H. Röttgering, ${ }^{3}$ S. Mandal, ${ }^{3}$ F. de Gasperin ${ }^{3}$ and M. Hoeft ${ }^{10}$ \\ ${ }^{1}$ Hamburger Sternwarte, University of Hamburg, Gojenbergsweg 112, D-21029 Hamburg, Germany \\ ${ }^{2}$ INAF/Istituto di Radioastronomia, Via P Gobetti 101, I-40129 Bologna, Italy \\ ${ }^{3}$ Leiden University, Rapenburg 70, NL-2311 EZ Leiden, the Netherlands \\ ${ }^{4}$ Harvard-Smithsonian Center for Astrophysics, 60 Garden Street, Cambridge, MA 02138, USA \\ ${ }^{5}$ Jodrell Bank Centre for Astrophysics, University of Manchester, Manchester M13 9PL, UK \\ ${ }^{6}$ Sydney Institute for Astronomy, School of Physics A28, The University of Sydney, NSW 2006, Australia \\ ${ }^{7}$ ARC Centre of Excellence for All-Sky Astrophysics (CAASTRO), The University of Sydney, NSW 2006, Australia \\ ${ }^{8}$ ASTRON, the Netherlands Institute for Radio Astronomy, Postbus 2, NL-7990 AA, Dwingeloo, the Netherlands \\ ${ }^{9}$ Kapteyn Astronomical Institute, University of Groningen, PO Box 800, NL-9700 AV Groningen, the Netherlands \\ ${ }^{10}$ Thüringer Landessternwarte, Sternwarte 5, D-07778 Tautenburg, Germany
}

Accepted 2017 September 29. Received 2017 September 20; in original form 2017 May 5

\begin{abstract}
Low-Frequency Array (LOFAR) observations at $144 \mathrm{MHz}$ have revealed large-scale radio sources in the unrelaxed galaxy cluster Abell 1132. The cluster hosts diffuse radio emission on scales of $\sim 650 \mathrm{kpc}$ near the cluster centre and a head-tail (HT) radio galaxy, extending up to $1 \mathrm{Mpc}$, south of the cluster centre. The central diffuse radio emission is not seen in NRAO VLA FIRST Survey, Westerbork Northern Sky Survey, nor in C \& D array VLA observations at $1.4 \mathrm{GHz}$, but is detected in our follow-up Giant Meterwave Radio Telescope (GMRT) observations at $325 \mathrm{MHz}$. Using LOFAR and GMRT data, we determine the spectral index of the central diffuse emission to be $\alpha=-1.75 \pm 0.19\left(S \propto \nu^{\alpha}\right)$. We classify this emission as an ultra-steep spectrum radio halo and discuss the possible implications for the physical origin of radio haloes. The HT radio galaxy shows narrow, collimated emission extending up to $1 \mathrm{Mpc}$ and another $300 \mathrm{kpc}$ of more diffuse, disturbed emission, giving a full projected linear size of $1.3 \mathrm{Mpc}$ - classifying it as a giant radio galaxy (GRG) and making it the longest HT found to date. The head of the GRG coincides with an elliptical galaxy (SDSS J105851.01+564308.5) belonging to Abell 1132. In our LOFAR image, there appears to be a connection between the radio halo and the GRG. The turbulence that may have produced the halo may have also affected the tail of the GRG. In turn, the GRG may have provided seed electrons for the radio halo.
\end{abstract}

Key words: galaxies: clusters: general - galaxies: clusters: individual: Abell 1132 -galaxies: clusters: intracluster medium - radio continuum: galaxies.

\section{INTRODUCTION}

Cluster-scale diffuse radio emission, in the form of radio haloes and radio relics, indicates the presence of large-scale magnetic fields and relativistic electrons within the intracluster medium (ICM). During a cluster merger, turbulence and shocks are produced in the

${ }^{\star}$ E-mail: amanda.wilber@hs.uni-hamburg.de (AW); mbrueggen@hs.unihamburg.de (MB)
ICM (e.g. Vazza, Brunetti \& Gheller 2009) and can lead to the reacceleration of mildly relativistic ICM electrons to ultra-relativistic speeds. The ultra-relativistic electrons (Lorentz factor $\gamma \gg 1000$ ) then interact with the ICM $B$-field (of the order of a few $\mu \mathrm{G}$ ) to produce synchrotron emission in the radio regime, and can lead to the formation of large-scale radio sources called haloes and relics (e.g. Kempner et al. 2004; see Feretti et al. 2012 for review). The origin of radio haloes and relics involves complex mechanisms, and further investigation is needed to understand how these mechanisms affect the physics of the ICM (see Brunetti \& Jones 2014 for review). 
Radio haloes are classified as diffuse radio emitters that fill the central regions of galaxy clusters, and are found to coincide with the thermal gas seen in X-ray observations. These radio structures are vast in size, usually extending up to $1 \mathrm{Mpc}$, and are typically characterized by a steep spectrum $(\alpha \lesssim-1)^{1}$ and low surface brightness $\left(\sim 1 \mu \mathrm{Jy} \operatorname{arcsec}^{-2}\right.$ at $1.4 \mathrm{GHz}$; e.g. Feretti et al. 2012). Two main models have been proposed for the origins of radio haloes: the hadronic model and the turbulent re-acceleration model. The hadronic model states that collisions between cosmic ray protons and thermal protons in the ICM would continuously produce the secondary electrons needed to generate radio halo emission at the cluster centre (Dennison 1980; Blasi \& Colafrancesco 1999; Enßlin et al. 2011). Hadronic collisions should also produce secondary gamma-ray photons, but the non-detection of galaxy clusters in the gamma-ray regime has constrained the contribution from secondary electrons to be subdominant (e.g. Ackermann et al. 2010, 2016; Jeltema \& Profumo 2011; Brunetti et al. 2012). The turbulent re-acceleration model states that mildly relativistic ICM electrons are re-accelerated to ultra-relativistic energies in situ during a cluster-sub-cluster merger (Brunetti et al. 2001; Petrosian 2001).

Cluster mergers are thought to produce turbulence that can accelerate cosmic rays and may amplify the magnetic fields in the ICM via the small-scale dynamo (Ryu et al. 2008; Keshet et al. 2010; Miniati \& Beresnyak 2016; Vazza et al. 2017). Indeed, studies that combine radio and X-ray data of galaxy clusters have suggested a causal link between merging activity of clusters and the occurrence of radio haloes (e.g. Cassano et al. 2013). Currently, turbulent re-acceleration is the favoured scenario for the origin of radio haloes, although several questions remain in identifying the seed source of mildly relativistic electrons within the ICM and in understanding the physics of cluster shocks and shock-induced turbulence. There are also a few outliers, where giant radio haloes are found in cool-core, non-merging clusters (e.g. Bonafede et al. 2014; Sommer et al. 2017), that challenge our present interpretation of ICM acceleration mechanisms.

A unique prediction of turbulent re-acceleration models is the existence of a large number of radio haloes with very steep spectra (Cassano, Brunetti \& Setti 2006; Brunetti et al. 2008). Steepspectrum haloes are produced when the turbulent re-acceleration rate is not efficient enough to accelerate electrons emitting at $\mathrm{GHz}$ frequencies, or during late evolutionary stages when turbulence is dissipated in the ICM (e.g. Cassano et al. 2012; Donnert et al. 2013). Searching for fading ultra-steep spectrum haloes may assist in clarifying the physical origins of radio haloes: the identification of breaks in the spectrum of haloes can be used to infer the efficiency of the mechanism that produces the emitting cosmic ray electrons (Thierbach, Klein \& Wielebinski 2003; Donnert et al. 2010a,b). A few cases of ultra-steep spectrum haloes have been found (e.g. Brunetti et al. 2008; Bonafede et al. 2012; Macario et al. 2013), but further sensitive low-frequency observations may be needed to reveal the population of ultra-steep haloes.

Active galactic nuclei (AGN) injection from individual cluster radio galaxies is one explanation for the large supply of mildly relativistic seed electrons needed for the turbulent re-acceleration that produces cluster-scale radio emission (such a connection has been established for certain cluster radio relics, as for example in van Weeren et al. 2017). Giant radio galaxies (GRGs), which are generally defined as radio galaxies with a linear projected size

\footnotetext{
${ }^{1}$ We define the spectral index, $\alpha$, where $S \propto v^{\alpha}$.
}

of $\gtrsim 0.7 \mathrm{Mpc}$ (e.g. Saripalli et al. 2005), can have a significant influence on their surrounding medium by supplying a large quantity of cosmic rays. Different scenarios may explain the formation and large extent of GRGs: the AGN may have been active for a very long time, the jets may be powerful enough to push emission out to large distances without much deterrence from the surrounding medium, and/or the surrounding medium could be much less dense as compared to the medium around typical radio galaxies (Kaiser \& Alexander 1999).

Tailed and bent-tailed radio galaxies are often found within the rich environments of galaxy clusters, and their jets give an indication of where cosmic ray electrons are being injected into the surrounding medium. These tailed sources have been typically categorized by their morphologies as seen in projection: as wide-angle-tail (WAT) when two radio jets, or plumes, are distinguishable, collimated, and open at an angle of $\lesssim 60^{\circ}$, or narrow-angle-tail (NAT) when the radio jets open in a very small angle such that they appear aligned on one side of the host galaxy or conjoined as a single tail [also referred to as head-tail (HT) radio galaxies] (e.g. Dehghan et al. 2014). It is generally thought that bent-tailed galaxies form when the jets experience ram pressure as the host galaxy moves through the ICM. A host galaxy travelling at high velocity may experience a ram pressure shock that aligns both radio jets behind the host's trajectory, leaving the radio source with a perceived NAT/HT morphology (Miley 1980). Of the known bent-tailed radio galaxies, only a small percentage are also GRGs. The longest HT discovered so far is in Abell 1314 with a projected linear size of $700 \mathrm{kpc}$ (Srivastava \& Singal 2016).

\subsection{Abell 1132}

Abell 1132 is a massive cluster $\left(5.87_{-0.23}^{+0.22} \times 10^{14} \mathrm{M}_{\odot}\right.$ from the Planck Collaboration XXIX 2014) that shows signs of merging (Cuciti et al. 2015), but has not shown diffuse radio emission in past VLA observations at $1.4 \mathrm{GHz}$ (Giovannini \& Feretti 2000). Abell 1132 is centred at RA, Dec. $10^{\mathrm{h}} 58^{\mathrm{m}} 25.8^{\mathrm{s}},+56^{\circ} 47^{\prime} 30^{\prime \prime}$ (equatorial, J2000.0) and located at a redshift of $z=0.1369$ (Struble \& Rood 1999). It contains several Fanaroff-Riley (FR) type-I radio galaxies (O'Dea \& Owen 1985), was covered by the NRAO VLA Sky Survey (NVSS; Condon et al. 1998) and has been observed by the Chandra X-ray Observatory.

Rudnick \& Lemmerman (2009) reported an extended HT source, $370 \mathrm{kpc}$ long, about $6^{\prime}$ south of the cluster centre using reprocessed data from the Westerbork Northern Sky Survey (WENSS; Rengelink et al. 1997). They noted that the head of this source is visible in NVSS and coincides with an elliptical galaxy ${ }^{2}$ belonging to the cluster.

In this paper, we report on Low-Frequency Array (LOFAR) observations and follow-up Giant Meterwave Radio Telescope (GMRT) observations of the galaxy cluster Abell 1132 and newly discovered extended radio emission. In the following section, details of our observations, data calibration, and imaging techniques are described. In Section 3, we show our LOFAR and GMRT images of the radio emission seen in Abell 1132, and in Section 4 we discuss how these images provide clues as to the morphology and possible origins of the detected emission. The scale at Abell 1132's redshift is $2.439 \mathrm{kpc} \operatorname{arcsec}^{-1}$ with the cosmological parameters $H_{0}=69.6$, $\Omega_{m}=0.286$ and $\Omega_{\Lambda}=0.714$, adopted hereafter.

${ }^{2} m R=16.78$ galaxy in SDSS (RA, Dec. $\left.=10^{\mathrm{h}} 58^{\mathrm{m}} 50.96^{\mathrm{s}},+56^{\circ} 43^{\prime} 08^{\prime \prime}\right)$, with a redshift of $z=0.138954 \pm 0.000162$. 
Table 1. Radio observations of A1132.

\begin{tabular}{lcc}
\hline Telescope & LOFAR & GMRT \\
\hline Observation ID & 544905 & $9101 / 9104$ \\
Pointing centre (RA, Dec.: J2000) & $11^{\mathrm{h}} 00^{\mathrm{m}} 20^{\mathrm{s}},+57^{\circ} 11^{\prime} 48^{\prime \prime}$ & $10^{\mathrm{h}} 58^{\mathrm{m}} 25.8^{\mathrm{s}}+56^{\circ} 47^{\prime} 30^{\prime \prime}$ \\
Observation date & $2016 \mathrm{September} 6$ & $2016 \mathrm{December} 30 / 31$ \\
Total on-source time & $8 \mathrm{~h}$ & $8 \mathrm{~h}$ \\
Flux calibrator & $3 \mathrm{C} 196$ & $3 \mathrm{C} 147$ and $3 \mathrm{C} 286$ \\
Total on-calibrator time & $10 \mathrm{~min}$ & $20 \mathrm{~min}$ \\
Central frequency & $144 \mathrm{MHz}$ & $325 \mathrm{MHz} / 610 \mathrm{MHz}$ \\
Bandwidth & $48 \mathrm{MHz}$ & $16 \mathrm{MHz} / 32 \mathrm{MHz}$ \\
\hline
\end{tabular}

\section{METHODS}

\subsection{LoTSS}

LOFAR is a low-frequency radio interferometer based in the Netherlands with additional international stations throughout Europe (van Haarlem et al. 2013). The array includes low-band and high-band antennas (LBA and HBA) that receive signals over a frequency range of 10-90 and 120-240 MHz, respectively. LOFAR's large field of view, compact core and its high sensitivity at low frequencies makes it the ideal instrument to study steep spectrum diffuse radio emission in galaxy clusters as well as GRGs with large angular diameter and low surface brightness.

The LOFAR Two-metre Sky Survey (LoTSS; Shimwell et al. 2017) Tier-1 has currently observed 14 per cent of the northern sky at $120-168 \mathrm{MHz}$ (as of 2017 July) with one of its main science goals to study galaxy clusters and search for cluster-scale radio emission. With a depth of $\sim 100 \mu \mathrm{Jy}$ beam $^{-1}$ at a resolution of $\sim 5 \operatorname{arcsec}$, this survey is particularly sensitive to steep spectrum radio emission, and thanks to the compact core of LOFAR it can detect radio objects with low surface brightness (see Shimwell et al. 2016, 2017, for details).

The observation of Abell 1132 was part of a standard Tier-1 survey observation, covering an area of $\sim 19 \mathrm{deg}^{2}$ centred on RA, Dec. $11^{\mathrm{h}} 00^{\mathrm{m}} 20^{\mathrm{s}},+57^{\circ} 11^{\prime} 48^{\prime \prime}$, made with the LOFAR Dutch HBA array. ${ }^{3}$ The north-western region of this field overlaps the Lockman hole (a region of space that has a low column density of hydrogen) which was also covered by LoTSS. Although Abell 1132 is visible in those observations (see Mahony et al. 2016 and Brienza et al. 2017 for details), here we provide a more detailed analysis of the radio emission seen in Abell 1132 with our more recent and more sensitive Tier-1 observation centred on Abell 1132. Our survey field was observed for $8 \mathrm{~h}$ to ensure sufficient $u v$-coverage. The observation covers a total bandwidth of $48 \mathrm{MHz}$ with a central frequency of $144 \mathrm{MHz}$. See Table 1 for observation details.

\subsection{LOFAR data reduction}

The standard data reduction for LoTSS data can be summarized into the following steps:

(i) Pre-facet calibration via Prefactor ${ }^{4}$ : Computes directionindependent solutions from observation of a standard calibrator and transfers these solutions to the target data. Performs an initial phase calibration for the target data using a global sky model. Produces preliminary images of the full field of view.

\footnotetext{
${ }^{3}$ The Dutch array consists of 24 core and 14 remote stations in the Netherlands.

${ }^{4}$ https://github.com/lofar-astron/prefactor
}

(ii) Facet calibration via FACTOR $^{5}$ (van Weeren et al. 2016): Performs direction-dependent calibration in multiple directions over the full bandwidth of target data. Corrects for ionospheric disturbances and beam errors. Produces high-resolution images.

In the following subsections, these steps will be elaborated upon for this particular observation.

\subsubsection{Pre-facet calibration}

The calibrator chosen for this observation was 3C196, a bright quasar (74 Jy according to the Scaife \& Heald (2012) absolute flux scale). The Prefactor pipeline was used to compute the amplitude gains, station clock offsets, station phase correlation offsets, and station differential total electron content (dTEC) from the standard calibrator data and apply all direction-independent solutions to the target data. ${ }^{6}$ After direction-independent solutions were transferred, an initial phase calibration was performed on the target field using a global sky model produced from the VLA Low-Frequency Sky Survey (VLSSr; Lane et al. 2012), WENSS (Rengelink et al. 1997) and NVSS (Condon et al. 1998). Data from the station CS030HBA were discarded (flagged) because it was not operational for most of the observation.

The Initial-Subtract step, as part of the pre-facet calibration pipeline, imaged the direction-independent calibrated target data in both high and low resolution using WSClean (Offringa et al. 2014). The pipeline first created an image at a resolution of $\sim 36 \operatorname{arcsec} \times 27$ arcsec and automatically detected and masked sources using the source detection software PYBDSF. ${ }^{7}$ The clean components of the masked sources were subtracted from the $u v$-data. Then an image was created at a resolution of $\sim 120 \operatorname{arcsec} \times 100$ arcsec where more extended sources were revealed, masked and also subtracted. The final result was a sky model with all high-resolution (compact sources) and low-resolution sources (extended sources) subtracted and a source-subtracted $u v$ data set, which was then used for direction-dependent calibration.

\subsubsection{Facet calibration}

Facet calibration is a direction-dependent calibration method for LOFAR, implemented by the FACTOR ${ }^{8}$ package (van Weeren et al. 2016). The first step is to tesselate the full field into multiple

\footnotetext{
${ }^{5}$ https://github.com/lofar-astron/factor

${ }^{6}$ dTEC solutions from the calibrator were not transferred to the target data since they correspond to the ionosphere and are therefore directiondependent.

${ }^{7}$ http://www.astron.nl/citt/pybdsf/

${ }^{8} \mathrm{http}: / /$ www.astron.nl/citt/facet-doc/
} 
facets. Each facet must have its own calibrator, designated by a square region within the facet. The calibrator region is chosen by default to be centred on a compact source at least $0.3 \mathrm{Jy}$ in flux density. The calibration region can be modified by the user to include multiple sources (increasing the calibrator flux density) and/or to include extended emission. To accurately calibrate a typical LoTSS widefield, up to 50 facet directions may be needed.

A process of self-calibration is conducted within the calibration region per facet, where several phase only and phase + amplitude self-calibration cycles are performed until there is convergence. The self-calibration solutions of a given calibrator region are then applied to its full facet, and the facet is imaged using WSClean. Each subsequent facet is imaged where all sources from prior facets are subtracted. Typically, facets are processed in order of calibrator brightness; this gradually decreases the effective noise in the $u v$ data. Facet images can be stitched together and primary-beamcorrected for a mosaic image of the full target field. For more details on facet calibration the reader is referred to van Weeren et al. (2016), Shimwell et al. (2016) and Williams et al. (2016).

FACTOR was run on the full $48 \mathrm{MHz}$ bandwidth for our observation. A total of 39 directions were designated and 15 bright and nearby facets were processed before processing the target facet containing the cluster Abell 1132. Initially our target calibration region included Abell 1132's complex cluster centre. The calibration in this region was inadequate since emission was being displaced and creating bright and negative artefacts. We decided instead to use the calibration solutions from the nearest facet, $\sim 0.25^{\circ}$ east of Abell 1132 's centre. The final image produced by FACTOR using WSClean has a beam size of $\sim 8 \operatorname{arcsec} \times 5 \operatorname{arcsec}$ with root mean square (rms) noise of $\sigma \approx 120 \mu \mathrm{Jy}_{\text {beam }}{ }^{-1}$.

The FACTOR-calibrated data were also imaged outside of FACTOR using CASA (Common Astronomy Software Applications; McMullin et al. 2007) tools. CASA CLEAN was used with various adjusted parameters ( $u v$-taper and Briggs' robust ${ }^{9}$ weighting schemes) so that diffuse emission would be properly masked and deconvolved. An increased outer $u v$-taper was used to bring out diffuse emission at lower resolutions.

\subsection{Chandra X-ray data reduction}

Abell 1132 was observed with the Chandra ACIS-I (ObsID: 13376) in 2011 Aug for $8 \mathrm{ks}$. We processed the Chandra data following Vikhlinin et al. 2005. ${ }^{10}$ This processing includes filtering of periods with elevated background by examining the light curves in the 6-12 keV band, the application of gain maps to calibrate photon energies, and corrections for the position-dependent charge transfer inefficiency. For the final exposure corrected image we used a pixel binning of $4\left(2 \operatorname{arcsec}\right.$ pixel $\left.^{-1}\right)$. The instrumental and sky background were subtracted. For more details the reader is referred to Vikhlinin et al. (2005).

To obtain the global temperature and luminosity within $R_{500}$ we fit the spectrum in XSPEC (v12.9, Arnaud 1996), extracting counts in the $0.7-7.0 \mathrm{keV}$ band. For $R_{500}$ we took a value $1.218 \mathrm{Mpc}$, derived from the mass of $5.9 \times 10^{14} \mathrm{M}_{\odot}$ (Planck Collaboration XXIX 2014). Compact sources were excluded from the fitting. The abundance was fixed to $0.3 \mathrm{Z}_{\odot}$ (from the abundance table of Anders $\&$ Grevesse 1989) and the redshift at $z=0.1366$. The hydrogen

\footnotetext{
${ }^{9} \mathrm{http} / / /$ www.aoc.nrao.edu/dissertations/dbriggs/

${ }^{10}$ We used CIAO v4.6 and CALDB v4.7.2.
}

column density, $N_{\mathrm{H}}$, was fixed to $6.29 \times 10^{19} \mathrm{~cm}^{-2}$, adopting the galactic value (atomic + molecular) from Willingale et al. (2013).

From the XSPEC fitting we determine a global temperature of $7.85_{-0.46}^{+0.46} \mathrm{keV}$ and a luminosity of $(4.4 \pm 0.1) \times 10^{44} \mathrm{erg} \mathrm{s}^{-1}$ in the $0.1-2.4 \mathrm{keV}$ energy band and $(2.7 \pm 0.1) \times 10^{44} \mathrm{erg} \mathrm{s}^{-1}$ in the $0.5-2.0 \mathrm{keV}$ energy band. The bolometric luminosity is $(10.6 \pm 0.3) \times 10^{44} \mathrm{erg} \mathrm{s}^{-1}$.

Abell 1132 is an unrelaxed, merging cluster according to its disturbed X-ray morphology revealed by Chandra, and it is one of the few merging systems belonging to samples of massive clusters without diffuse radio emission seen at higher frequencies (Cuciti et al. 2015). Given the $L_{1}-T_{1}$ relation (BCES $(Y \mid X)$ fitting) in table 2 of Pratt et al. (2009), the global temperature we measure $\left(7.85_{-0.46}^{+0.46} \mathrm{keV}\right)$ would correspond to a luminosity of $(2.2 \pm 0.1) \times 10^{45} \mathrm{erg} \mathrm{s}^{-1}$ in the $0.1-2.4 \mathrm{keV}$ energy band. This is an order of magnitude higher than our measured luminosity, thus fitting into the evidence that suggests this cluster is a merger.

\subsection{GMRT data reduction}

Follow-up GMRT observations of Abell 1132 at 325 and $610 \mathrm{MHz}$ were performed on 2016 December 30 and 31 (see Table 1 for observation details). The GMRT data were processed using the SPAM pipeline (see Intema et al. 2017, for details). Our images with the highest resolution and lowest noise were produced in AIPS (Astronomical Image Processing System; Wells 1985). These data were also imaged with CASA CLEAN interactively with various adjusted parameters so that diffuse emission would be properly masked and de-convolved.

\section{RESULTS}

Observations of Abell 1132, as part of LoTSS Tier-1 (Shimwell et al. 2017), reveal several previously unknown regions of radio emission associated with the cluster. In Fig. 1, we present our overview image of Abell 1132 where LOFAR radio emission in high and low resolution and Chandra X-ray emission are overlaid on an optical image from the Sloan Digital Sky Survey (SDSS). The cluster hosts diffuse radio emission near the cluster centre, slightly offset from the X-ray emission, and a giant HT/NAT radio galaxy south of the cluster centre with collimated emission to the east and diffuse emission to the west. A WAT radio galaxy, lying $\sim 0.2^{\circ}(1.8 \mathrm{Mpc})$ west of the cluster centre, is also visible in our LoTSS observation (as shown in Fig. 2). Follow-up GMRT observations also show a significant portion of the HT radio galaxy and the full WAT radio galaxy at 325 and $610 \mathrm{MHz}$ (see Fig. 3). The heads of the two tailed radio galaxies coincide with elliptical galaxies belonging to the cluster: SDSS J105851.01+564308.5 at $z \approx$ 0.139 (HT) and SDSS J105702.79+564503.1 at $z \approx 0.136$ (WAT) (see Fig. 4; redshifts taken from the SDSS Data Release 2 from Abazajian et al. 2004).

\subsection{Radio halo emission in Abell 1132}

Cluster-scale diffuse emission, characteristic of a radio halo, is visible in both the LOFAR image at $144 \mathrm{MHz}$ and the GMRT image at $325 \mathrm{MHz}$ of Abell 1132. Fig. 2 shows our low-resolution LOFAR image with our low-resolution GMRT $325 \mathrm{MHz}$ image contours overlaid. In Fig. 2, the halo appears more extensive in the LOFAR image, extending towards and possibly connecting to the diffuse emission of the giant southern HT galaxy. 


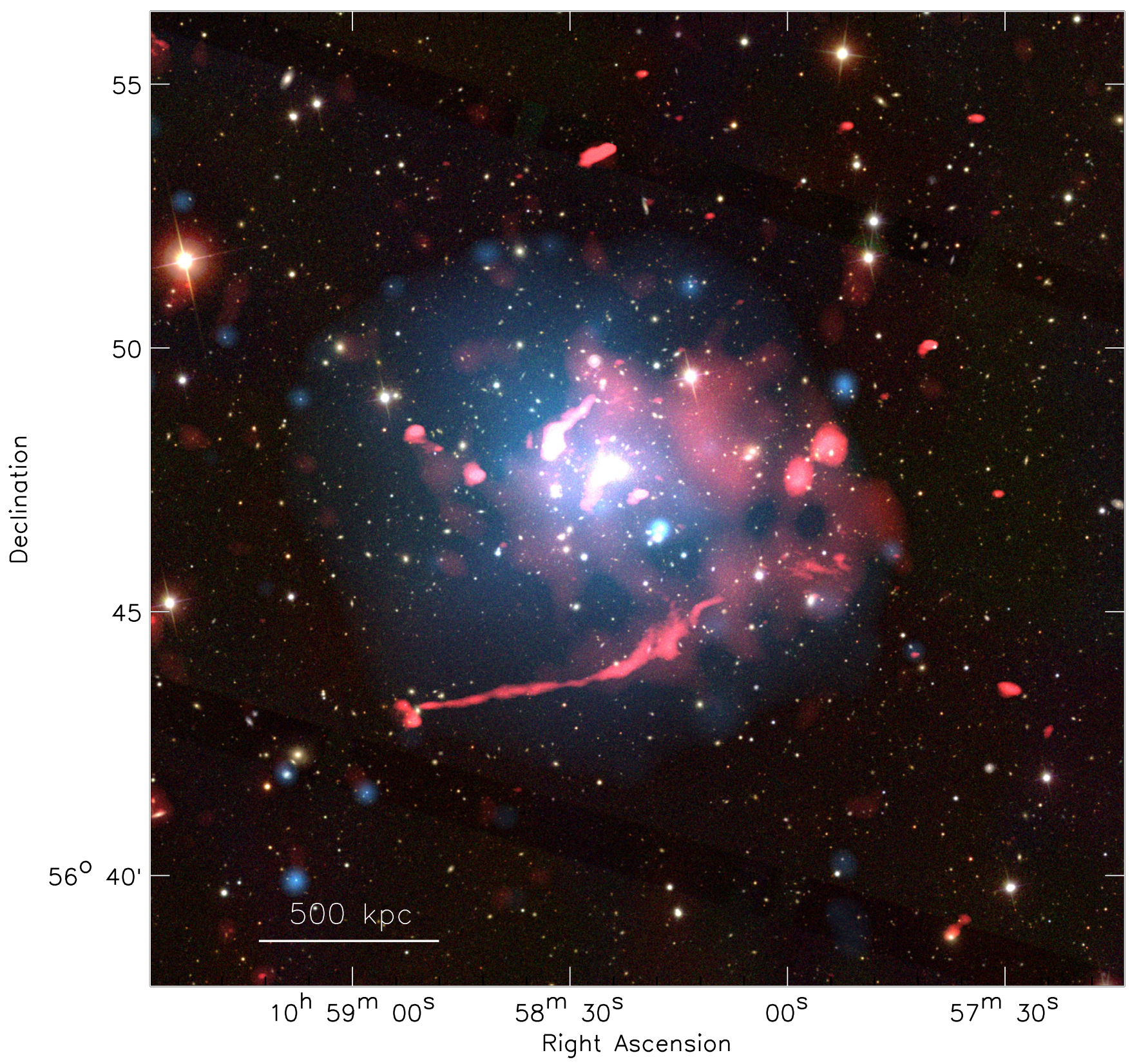

Figure 1. Overview image of Abell 1132: LOFAR high-resolution and low-resolution emission are both shown in red. LOFAR high-resolution emission is imaged with a beam size of $\sim 8 \operatorname{arcsec} \times 5$ arcsec and rms noise of $120 \mu \mathrm{Jy}$ beam ${ }^{-1}$. LOFAR low-resolution diffuse emission (imaged after performing a subtraction of compact sources, as explained in Section 3.1) is imaged with a beam size of $\sim 30 \operatorname{arcsec} \times 26$ arcsec and rms noise of $350 \mu \mathrm{Jy}$ beam ${ }^{-1}$. Chandra $\mathrm{X}$-ray emission is in blue. Radio and X-ray emission are overlaid on optical SDSS $g, r, i$ images. Diffuse radio emission is present near the centre of the cluster, as well as in the length of the southern HT GRG. The central diffuse radio emission, characteristic of a radio halo, is offset from the centre of the X-ray emission by $\sim 200 \mathrm{kpc}$. There also appears to be a connection between the radio halo and the diffuse radio emission of the GRG tail.

As seen in Fig. 2, there are several bright and extended FRI galaxies near the cluster centre. We performed a compact-sourcesubtraction on our 144 and $325 \mathrm{MHz}$ data to better image the diffuse emission and eliminate contamination from the central radio galaxies. Since the halo is detected as diffuse emission on the scale of $\sim 500-700 \mathrm{kpc}$, we subtracted compact sources corresponding to emission spanning less than $500 \mathrm{kpc}$. At Abell 1132's redshift, this corresponds to visibility data greater than $1000 \lambda$ in the $u v$-plane. We made an image in CASA CLEAN with a $u v$-cut below $1000 \lambda$ and an outer $u v$-taper of $10 \mathrm{arcsec}$, and subtracted the model component from the $u v$-data using CASA tools $\mathrm{FT}^{11}$ and UVSUB. We then re-imaged the source-subtracted data sets with their full $u v$ range $^{12}$ to bring out extended emission (see Fig. 6: left). The residual emission from the central galaxies is $<1$ percent of their flux densities.

\footnotetext{
${ }^{11}$ We used our own CASA task called FTW which includes the widefield w-projection parameter.

$12>80 \lambda$ for $144 \mathrm{MHz}$ and $>100 \lambda$ for $325 \mathrm{MHz}$.
} 


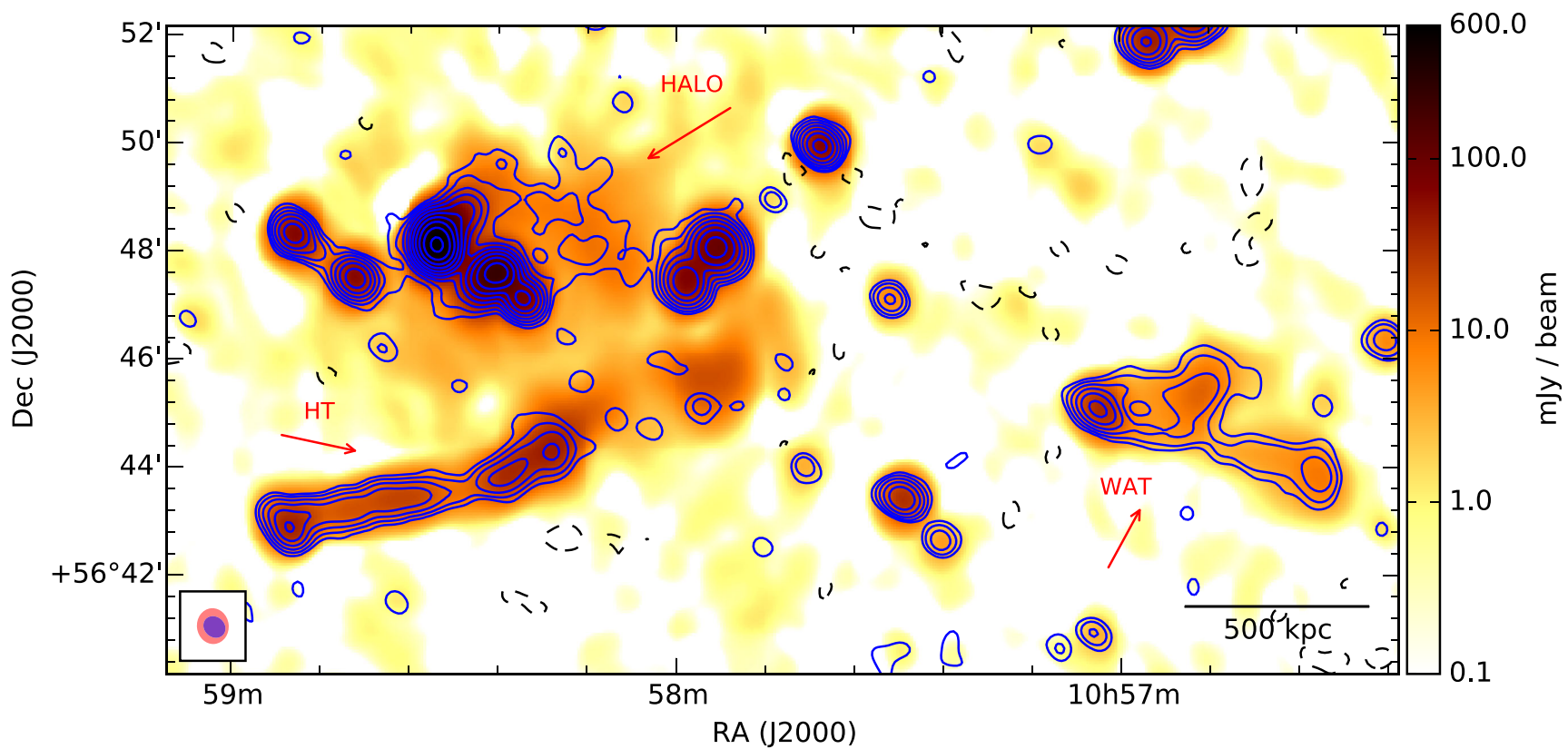

Figure 2. Abell 1132: $144 \mathrm{MHz}$ LOFAR low-resolution emission is shown in colour (on a logarithmic scale) with $325 \mathrm{MHz}$ GMRT low-resolution contours overlaid in blue. LOFAR and GMRT emission are imaged in CASA CLEAN with $u v$-taper 25 arcsec and Briggs' robust 0 , with respective rms noise of 400 and $220 \mu \mathrm{Jy}$ beam $^{-1}$. Contours are $\sigma \times[-2,2,4,8,16,32,128,256,512,1024]$. Beam size is designated by the red (LOFAR $\sim 40$ arcsec $\times 35$ arcsec) and blue (GMRT $\sim 26$ arcsec $\times 22$ arcsec) ellipses. Diffuse emission is present near the cluster centre as well as in the westward portion of the giant HT. A WAT is visible $\sim 0.2^{\circ}(1.8 \mathrm{Mpc})$ west of the cluster centre.
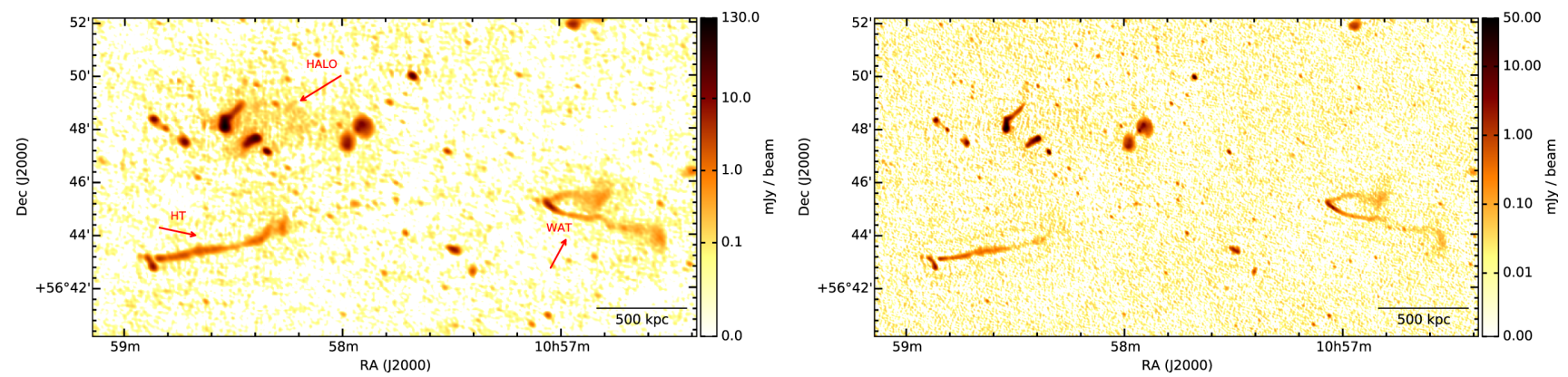

Figure 3. Left: GMRT image at $325 \mathrm{MHz}$ with a resolution of $\sim 10 \operatorname{arcsec} \times 7 \operatorname{arcsec}$ and $\mathrm{rms}$ noise of $\sigma \approx 45 \mu \mathrm{Jy}$ beam $^{-1}$. Right: GMRT image at $610 \mathrm{MHz}$ with a resolution of $\sim 6 \operatorname{arcsec} \times 4$ arcsec and rms noise of $\sigma \approx 20 \mu \mathrm{Jy}$ beam ${ }^{-1}$. GMRT data were calibrated via the SPAM pipeline and imaged in AIPS with Briggs' robust -1 . Both GMRT images show a significant portion of the HT radio galaxy and the full WAT radio galaxy. The radio halo is partially visible at $325 \mathrm{MHz}$.
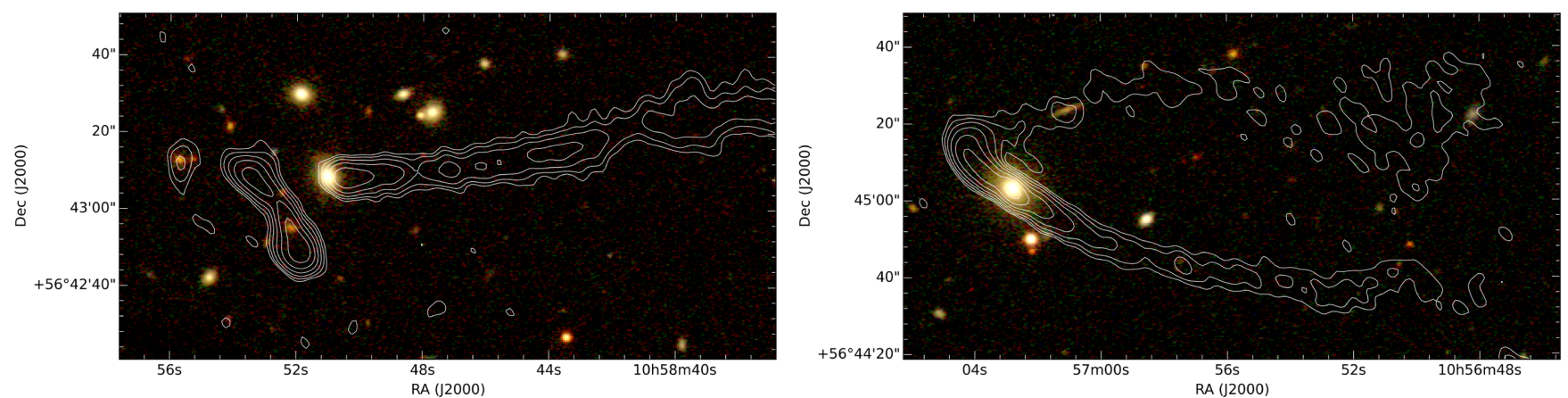

Figure 4. SDDS $g, r, i$ optical images overlaid with GMRT contours at $610 \mathrm{MHz}$. The levels are $[3,6,12,24,48,96] \times \sigma$ where $^{2}=20 \mu \mathrm{Jy}$ beam ${ }^{-1}$. Left: the optical source associated with the HT GRG is the elliptical galaxy SDSS J105851.01+564308.5 at a redshift of $z \approx 0.139$, within Abell 1132. The double source to the east of the HT is likely associated with the background galaxy SDSS J105852.18+564255.3 at a redshift of $z \approx 0.496$. Right: the optical source associated with the WAT is the elliptical galaxy SDSS J105702.79+564503.1 at a redshift of $z \approx 0.136$, within Abell 1132 . 


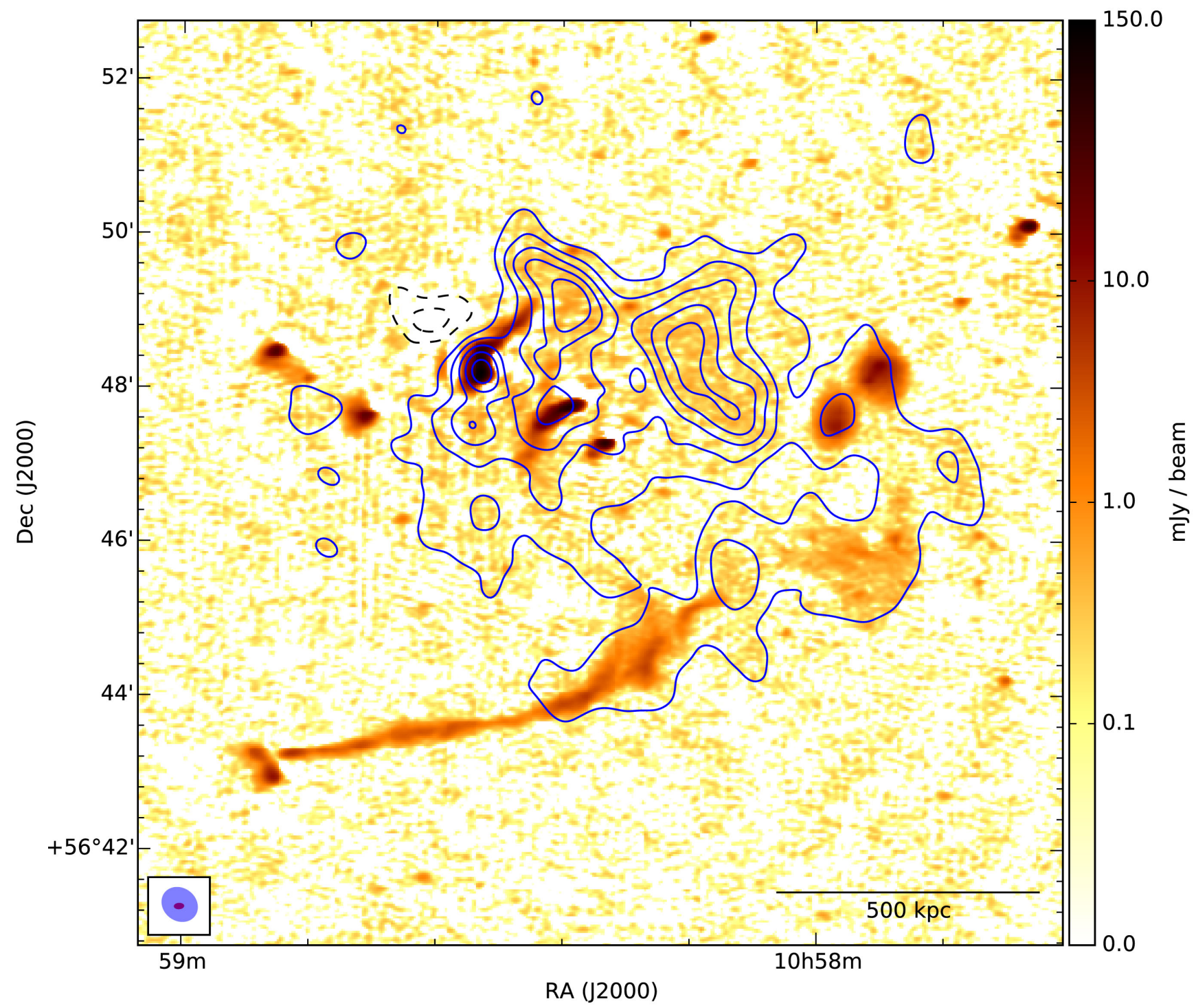

Figure 5. Our FACTOR image: LOFAR high-resolution emission is shown in colour (on a logarithmic scale), imaged with a beam size of $\sim 8$ arcsec $\times 5$ arcsec and rms noise of $120 \mu \mathrm{Jy}$ beam $^{-1}$, with LOFAR low-resolution diffuse emission contours overlaid in blue. LOFAR diffuse emission is imaged in CASA CLEAN with an outer $u v$-taper of 20 arcsec and Briggs' robust 0 after subtracting compact sources imaged above a $u v$-range of 1000ג (as explained in Section 3.1). rms noise of LOFAR low-resolution diffuse emission is $\sigma=350 \mu \mathrm{Jy}_{\text {beam }}{ }^{-1}$ and the contour levels are $[-6,-3,3,6,9,12,15] \times \sigma$. Beam size is designated by the red (high resolution $\sim 8 \operatorname{arcsec} \times 5 \operatorname{arcsec}$ ) and blue (low resolution $\sim 30 \operatorname{arcsec} \times 26 \operatorname{arcsec}$ ) ellipses.

LOFAR diffuse emission, after compact-source-subtraction, is shown in red in Fig. 1 and as contours overlaid on our FACTOR image in Fig. 5. It is apparent in Fig. 5 that there is a hole (negative artefact) to the north-east of the brightest cluster galaxy. The negative artefact likely occurred because there was imperfect calibration, modelling and subtraction of the brightest cluster galaxy, as it has no prior model and it is embedded in diffuse halo emission. In LOFAR facet-calibrated images, negative artefacts or negative bowls, often occur near bright sources that have not been previously modelled. This hole appears to be the only one in the cluster field and is on a relatively small scale. The negative artefact remains after the compact-source-subtraction, and is indicated by negative contours (dashed) in Fig. 5. It is possible that the halo emission extends within this region, but future low-frequency observations would be needed for confirmation.
Diffuse emission, after compact-source-subtraction, at 144 and $325 \mathrm{MHz}$ are shown together in Fig. 6. The size and morphology of the halo is comparable in both the LOFAR and GMRT images, however, the LOFAR image of the halo shows some additional, weaker emission to the south, possibly connecting to the diffuse emission in the giant HT. The bulk of the detected diffuse emission lies slightly west of the cluster centre, exhibiting a subtle offset $(\sim 200 \mathrm{kpc}$ ) from the centre of the Chandra X-ray emission. The halo takes on a roughly elliptical shape, shorter in the north-south direction and elongated from south-east to north-west, with a major axis of $\sim 750 \mathrm{kpc}$ and minor axis of $\sim 570 \mathrm{kpc}$, as seen by LOFAR. The surface brightness of the halo (after subtraction of central galaxies) within $3 \sigma$ contours is $\sim 0.8 \mu \mathrm{Jy} \operatorname{arcsec}^{-2}$ at $144 \mathrm{MHz}$ with $\sigma=350 \mu \mathrm{Jy}$ beam $^{-1}$ and a beam size of $30 \operatorname{arcsec} \times 26 \operatorname{arcsec}$ (see Fig. 5). 

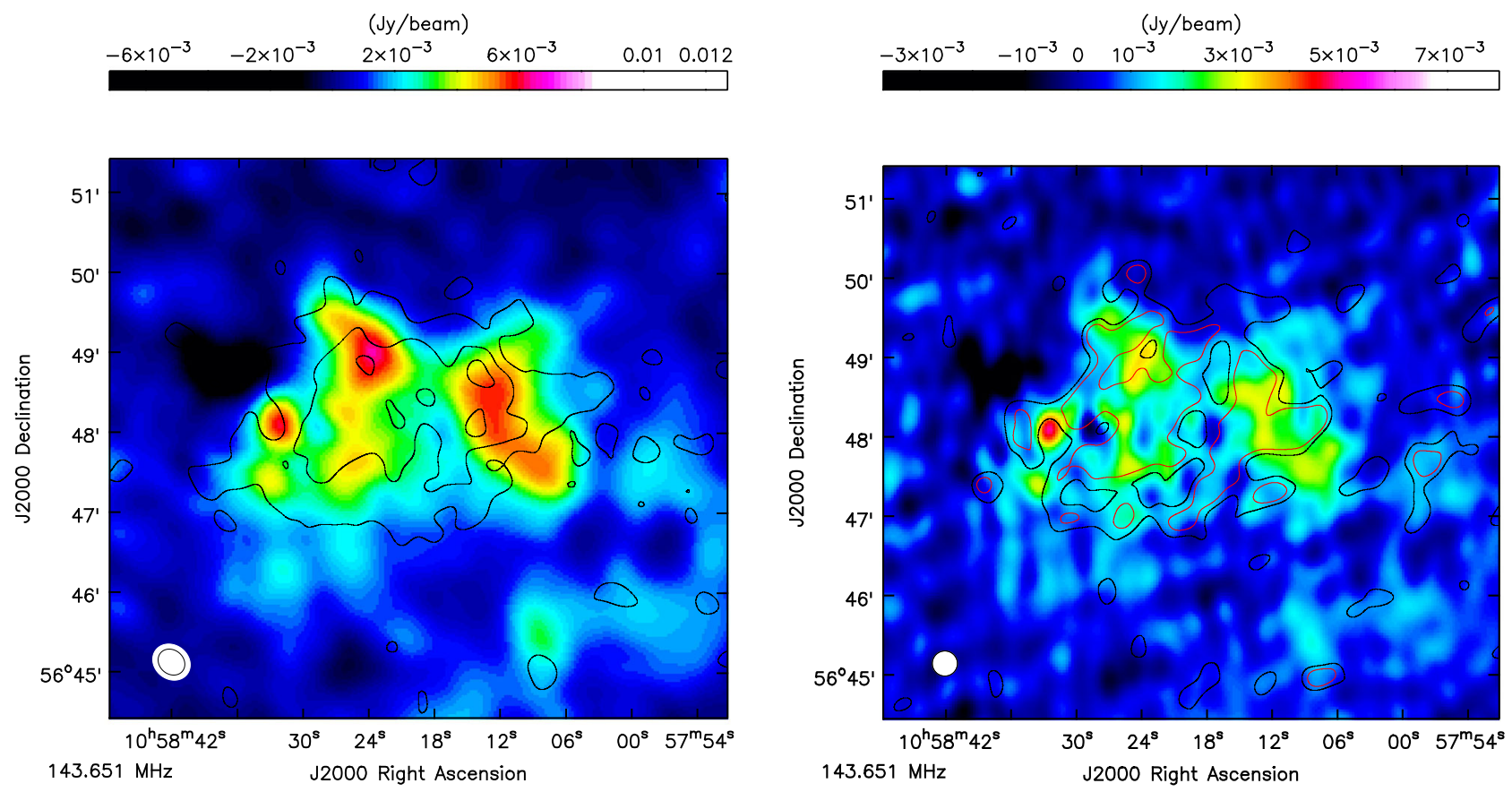

Figure 6. LOFAR low-resolution diffuse emission after subtraction of compact sources (as explained in Section 3.1.1) is shown in colour and GMRT $325 \mathrm{MHz}$ low-resolution diffuse emission after subtraction of compact sources is show as contours overlaid. Left: Our optimal image of the diffuse component in Abell 1132. Both LOFAR and GMRT compact-source-subtracted data sets were imaged in CASA CLEAN with an outer $u v$-taper of 20 arcsec and Briggs' robust 0. Contours represent $[3,6,9] \times \sigma$ at $325 \mathrm{MHz}$ where $\sigma=75 \mu \mathrm{Jy}_{\text {beam }}{ }^{-1}$. Right: Our uniform-weighted image of the diffuse emission in Abell 1132 . Both

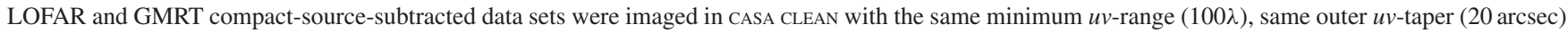
and uniform weighting, and were re-gridded and smoothed to the same beam $(19 \mathrm{arcsec})$. Black contours represent $2 \sigma$ and red contours represent $3 \sigma$ at $325 \mathrm{MHz}$ where $\sigma=130 \mu \mathrm{Jy}_{\text {beam }}{ }^{-1}$. The spectral index estimates stated in Section 3.1.1 were calculated by comparing the measured flux density within the $2 \sigma$ and $3 \sigma$ regions at $325 \mathrm{MHz}$. The red $3 \sigma$ contours at $325 \mathrm{MHz}$ define an east and west region of the halo, where separate measurements were taken. The final estimate for the spectral index is an average of the values calculated within the $2 \sigma$ region and the two $3 \sigma$ regions. The residual flux of the brightest cluster galaxy was not included as measured flux in these regions.

We produced GMRT images at $610 \mathrm{MHz}$ tapered to 30 arcsec resolution to enhance diffuse emission, but the radio halo is not detected above $2 \sigma$ where $\sigma \approx 100 \mu \mathrm{Jy}_{\text {beam }}{ }^{-1}$. Archival VLA observations performed in D array were retrieved, reduced and re-imaged, but diffuse emission near the cluster centre is not detected above $2 \sigma$ where $\sigma \approx 250 \mu \mathrm{Jy}_{\text {beam }^{-1}}$.

\subsubsection{Halo spectral index estimate}

We estimate the spectral index of the halo by imaging the compactsource-subtracted data sets at 144 and $325 \mathrm{MHz}$ in CASA CLEAN with

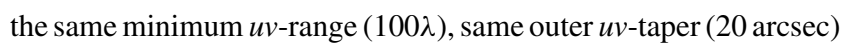
and uniform weighting, and compare the flux densities within the same region after re-gridding ${ }^{13}$ and smoothing to the same beam size $(19 \operatorname{arcsec} \times 19 \operatorname{arcsec})$. In a region indicated by $2 \sigma$ contours at $325 \mathrm{MHz}$ where $\sigma=130 \mu \mathrm{Jy}$ beam $^{-1}$, the spectral index is $\alpha=-1.80 \pm 0.18$. In a region indicated by $3 \sigma$ contours at $325 \mathrm{MHz}$ (east portion of halo), the spectral index is $\alpha=-1.71 \pm 0.19$, and in another region indicated by $3 \sigma$ contours at $325 \mathrm{MHz}$ (west portion

\footnotetext{
${ }^{13}$ We shifted the LOFAR map by -2 pixels in $X$-direction and -3 pixels in $Y$ direction to correct for an astrometric offset. This pixel shift was determined by comparing the high-resolution LOFAR and GMRT maps imaged with the same settings and convolved to the same beam (11 arcsec) and calculating the offset of the maximum pixel of several point sources near the cluster centre.
}

of halo) the spectral index is $\alpha=-1.74 \pm 0.20$ (see the regions as contours in Fig. 6: right). Therefore, we give an average spectral index estimate of $\alpha=-1.75 \pm 0.19$ and classify this radio halo as ultra-steep. With a spectral index of $\alpha=-1.75$, the surface brightness of the halo emission would be $\sim 1.3 \mu \mathrm{Jy} \operatorname{arcsec}^{-2}$ at $1.4 \mathrm{GHz}$, and considering a 15 percent error ${ }^{14}$ in our total measured flux at $144 \mathrm{MHz}$ and an error of \pm 0.19 in the spectral index, the radio power at $1.4 \mathrm{GHz}$ is determined to be $P_{1.4}=(1.66 \pm 0.76) \times 10^{23}$ $\mathrm{W} \mathrm{Hz}{ }^{-1}$.

It has been found that the radio power of haloes correlate with the X-ray luminosity of the host cluster (Brunetti et al. 2007, 2009; Cassano et al. 2013; Yuan, Han \& Wen 2015). In Fig. 7, we plot the radio power at $1.4 \mathrm{GHz}$ versus the Planck cluster mass $M_{500}$ for a sample of radio haloes and include Abell 1132's halo, indicated by the red star. The halo is not only ultra-steep but also extremely weak: the plot in Fig. 7 shows Abell 1132's halo lying well below the correlation line. It is possible that this ultra-steep halo sets an unprecedented record for the weakest halo discovered so far. The fact that it is so steep and weak, as seen at low frequency, is consistent with the non-detection of diffuse emission at $1.4 \mathrm{GHz}$.

\footnotetext{
${ }^{14}$ We approximate the error in the halo's flux by assuming a 10 percent error from FACTOR calibration, modelling and imaging (based on experience) and introducing a 5 percent error from the contamination of the residual emission of subtracted central galaxies.
} 


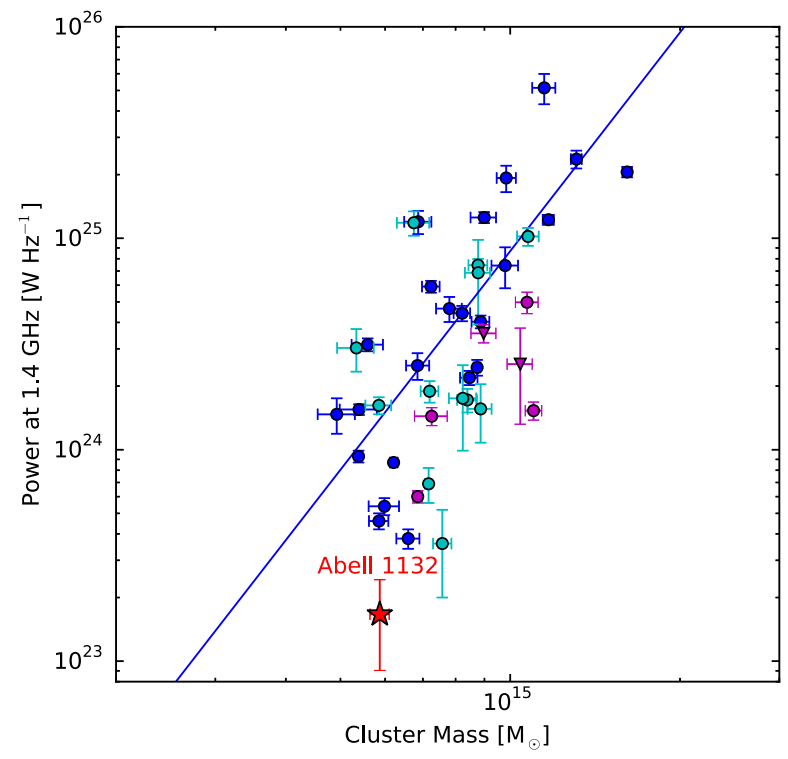

Figure 7. A sample of radio haloes plotted by their radio power at $1.4 \mathrm{GHz}$ versus their cluster mass ( $M_{500}$ - as determined from Planck observations). The sample of haloes and their correlation is reproduced from Martinez Aviles et al. (2016). Haloes with flux measured at $1.4 \mathrm{GHz}$ are marked by blue circles and their derived fit is shown as a blue line. Cyan circles represent haloes with flux measured at frequencies other than 1.4 GHz. Magenta circles represent ultra-steep haloes, and magenta triangles represent ultrasteep haloes with flux measured at frequencies other than $1.4 \mathrm{GHz}$. Abell 1132 is marked by the red star, and falls well below the correlation line as well as below all the haloes in this sample.

\subsection{Giant radio galaxy: head-tail}

The GRG is a prominent feature of the radio emission from the cluster, exhibiting long and narrow emission with a projected linear size of $1.3 \mathrm{Mpc}$. The head of this emission coincides with an elliptical galaxy $^{2}$ near the same redshift as Abell 1132 (see Fig. 4). The giant tail, as seen by LOFAR, is the same HT source as seen in NVSS and in reprocessed WENSS data, but there the tail is only detected to be $370 \mathrm{kpc}$ long (Rudnick \& Lemmerman 2009). The GRG appears to be one-sided, since only one jet is visible. It is likely that the jets have joined into a single tail aligned behind the trajectory of the galaxy as it has moved west-to-east. The giant tail in Abell 1132 has similar physical characteristics to the tail in Abell 2256 (Owen et al. 2014), however, it is more than twice as long as the tail in Abell 2256. If the host galaxy was moving at the sound speed of the cluster, $\sim 1000 \mathrm{~km} \mathrm{~s}^{-1}$, then it is possible that the AGN has been active for $\sim 1 \mathrm{Gyr}$, which is much greater than typical AGN life cycles (of the order of a few Myr).

The GRG has travelled from west-to-east through the cluster outskirts, leaving the observed tail as a trail of AGN emission, but its vast extent challenges the fact that the tail electrons $1 \mathrm{Mpc}$ and further from the AGN head should no longer be emitting. The separation in the tail at $1 \mathrm{Mpc}$, where the emission becomes more diffuse (see Figs 1 and 5), is interesting: here, dormant tail electrons may have been disrupted and re-accelerated, leading to a re-brightening that gives rise to the additional $300 \mathrm{kpc}$ of diffuse emission (de Gasperin et al. 2017 reports a similar re-brightening of dormant tail electrons from a WAT radio galaxy within the massive merging cluster Abell 1033 and attributes it to 'gentle re-energization'). It also appears that the radio halo connects to the diffuse portion of the tail (see Fig. 5). A connection between the halo and tail may give an indication of where the seed particles needed for turbulent re-acceleration come from. However, a question remains in how the length of the tail up to $1 \mathrm{Mpc}$ could retain its collimated form if it has been affected by merger turbulence. Since the diffuse portion is not visible in the GMRT images at 325 or $610 \mathrm{MHz}$ (Fig. 3), it is likely to be a very steep and very weak emission. If the diffuse portion has been re-accelerated, its emission should have a slightly flatter spectral index than the steepest part of the collimated portion.

In Fig. 8, a spectral index map of LOFAR and GMRT images aids in classifying the HT galaxy. The spectrum steepens along the length of the tail, with a spectral index of $\alpha \approx-2$ at a distance of $\sim 800 \mathrm{kpc}$ from the head. At further distances only LOFAR detects emission, hence a spectral index could not be determined for the detached portion of the tail beyond $1 \mathrm{Mpc}$. An upper limit can be placed for the detached, more diffuse portion by comparing the mean flux at $144 \mathrm{MHz}$ to $2 \sigma$ at 325 and $610 \mathrm{MHz}$ (see caption of Fig. 8 for values of $\sigma$ ). The upper limit of the spectral index is $\alpha<-2.3$ for $325 \mathrm{MHz}$ and $\alpha<-1.7$ for $610 \mathrm{MHz}$.

The WAT to the west of the cluster centre (see Figs 2 and 3) follows a similar trajectory to the HT. It appears to be moving from west-to-east on the outskirts of the cluster, leaving two tails of AGN emission. The longer tail has a projected size of $\sim 650 \mathrm{kpc}$ and the shorter tail has a projected size of $\sim 450 \mathrm{kpc}$.

\section{DISCUSSION AND CONCLUSIONS}

The discovery of this radio halo in Abell 1132 demonstrates LOFAR's potential to detect weak, steep-spectrum emission on large scales. The steep-spectrum halo in Abell 1132 is noteworthy for three reasons:

(i) with a spectral index of $\alpha=-1.75 \pm 0.19$ it is one of the steepest haloes detected to date;

(ii) with a size of $\sim 650 \mathrm{kpc}$ and radio power of $P_{1.4}=(1.66 \pm 0.76) \times 10^{23} \mathrm{~W} \mathrm{~Hz}^{-1}$ the halo is smaller than usual and it is remarkably faint;

(iii) the halo is $\sim 200 \mathrm{kpc}$ offset from the X-ray emission of the cluster.

A few other ultra-steep haloes include $\alpha \approx-2.1$ in A521 (Brunetti et al. 2008), $\alpha \approx-1.7$ to -1.8 in A697 (Macario et al. 2010), and A1682 (Macario et al. 2013). Steep-spectrum radio haloes challenge hadronic models because energy arguments rule out the possibility that very steep haloes with $\alpha \sim-1.5$ to -2 are produced by cosmic ray electrons that follow power laws in momentum (Pfrommer \& Enßlin 2004; Brunetti et al. 2008). However, turbulent re-acceleration models do predict a large population of steepspectrum haloes, which exhibit a break in their electron spectra near energies of a few $\mathrm{GeV}$ (Brunetti et al. 2004, 2008). Most radio haloes known today, discovered at higher radio frequencies $(1.4 \mathrm{GHz})$, are produced by mergers between the most massive galaxy clusters. According to the turbulent re-acceleration model, radio haloes with much steeper spectra should be produced by less energetic, more frequent mergers. Hence, the bulk of radio haloes may have yet to be discovered because they are only visible at low frequencies. Given its sensitivity to diffuse emission with low surface brightness, LOFAR will be a valuable tool to reveal this population.

In hadronic models the radio emission of the halo should also roughly follow the X-ray surface brightness, as the X-ray emission traces the thermal ICM that provides the targets for the hadronic collisions. Clearly, this is not the case in the halo in Abell 1132. The fact that the halo is smaller and fainter also supports a turbulent re-acceleration origin of haloes, as the steeper-spectrum haloes are produced by older populations of relativistic electrons that can no 


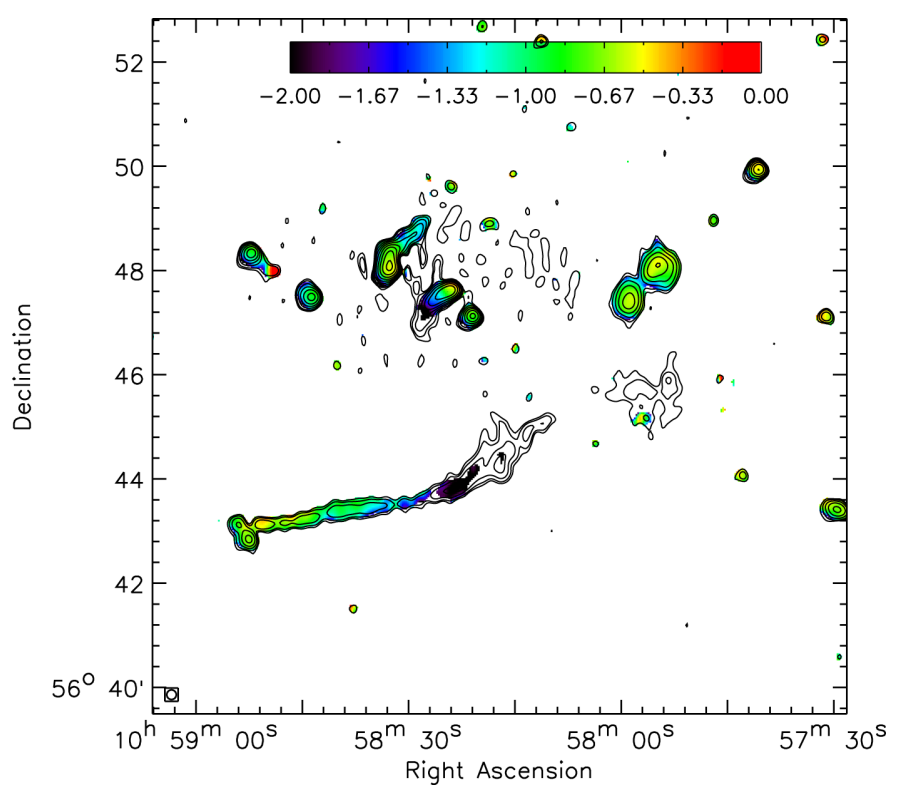

Figure 8. Spectral index map of the giant HT galaxy using emission cut above $3 \sigma$ from LOFAR at $144 \mathrm{MHz}$ and GMRT at 325 and $610 \mathrm{MHz}$. LOFAR and GMRT emission are imaged in CASA CLEAN with an outer $u v$-taper of $10 \operatorname{arcsec}$, uniform weighting and a minimum $u v$-range of $200 \lambda$ with rms noise of $\sigma_{140}=300 \mu \mathrm{Jy}_{\text {beam }}{ }^{-1}, \sigma_{325}=100 \mu \mathrm{Jy}$ beam $^{-1}$ and $\sigma_{610}=60 \mu \mathrm{Jy}_{\text {beam }}{ }^{-1}$. All images were smoothed to the same beam size $(11 \operatorname{arcsec} \times 11 \mathrm{arcsec})$. Maps at 144 and $325 \mathrm{MHz}$ were re-gridded to the map at $610 \mathrm{MHz}^{13}$. LOFAR emission is also shown as black contours with levels $\sigma_{140} \times[3.45,6,12,24,48,96$, $192,384,768]$.

longer sustain a luminous halo. Hence, we may be witnessing a radio halo that is transitioning into an 'off' state. It would be interesting to determine the dynamical status of the cluster merger using optical spectroscopy, in order to relate the radio properties of the halo to the phase and energetics of the cluster merger. With much better spectral capabilities than the current X-ray telescopes, the future Xray observatory ATHENA may be able to probe turbulence within Abell 1132's ICM.

The discovery of the radio halo and GRG found together in one cluster has raised the question about a possible connection. The presence of a radio halo suggests that the cluster has recently undergone a merger, but it is unclear whether the merger has affected the emission of the GRG. Near the GRG-head the emission appears to be mostly undisturbed, very narrow and collimated, but the furthest emission of the tail is more diffuse. It may be possible that the $1.3 \mathrm{Mpc}$ tail is visible because it has been re-accelerated by turbulent merger activity. A spectral index measurement of this disturbed portion as compared to the collimated portion would reveal the age of this emission and clarify whether it has been re-accelerated. This cluster is a prime target for studying merger mechanisms and the re-acceleration of dormant electrons.

\section{ACKNOWLEDGEMENTS}

This work was supported by the Deutsche Forschungsgemeinschaft (DFG) through the Collaborative Research Centre SFB 676 'Particles, Strings and the Early Universe', project C2. LOFAR, the Low-Frequency Array designed and constructed by ASTRON, has facilities in several countries, that are owned by various parties (each with their own funding sources), and that are collectively operated by the International LOFAR Telescope (ILT) foundation under a joint scientific policy. The LOFAR software and dedicated reduction packages on https://github.com/apmechev/GRID_LRT were deployed on the e-infrastructure by the LOFAR e-infragroup, consisting of J. B. R. Oonk (ASTRON \& Leiden Observatory),
A. P. Mechev (Leiden Observatory) and T. Shimwell (Leiden Observatory) with support from N. Danezi (SURFsara) and C. Schrijvers (SURFsara). This work has made use of the Dutch national e-infrastructure with the support of SURF Cooperative through grant e-infra160022. We thank the staff of the GMRT that made these observations possible. GMRT is run by the National Centre for Radio Astrophysics of the Tata Institute of Fundamental Research. This research has made use of the NASA/IPAC Extragalactic Data Base (NED) which is operated by the JPL, California Institute of Technology under contract with the National Aeronautics and Space administration. TS acknowledges support from the ERC Advanced Investigator programme NewClusters 321271. FA-S acknowledges support from Chandra grant GO3-14131X. EKM acknowledges support from the Australian Research Council Centre of Excellence for All-sky Astrophysics (CAASTRO), through project number CE110001020. RM gratefully acknowledges support from the European Research Council under the European Union's Seventh Framework Programme (FP/2007-2013) ERC Advanced Grant RADIOLIFE-320745. AOC gratefully acknowledges support from the European Research Council under grant ERC-2012-StG-307215 LODESTONE. We also thank A. Botteon (Università di Bologna) and G. Kokotanekov (University of Amsterdam) for their helpful comments.

\section{REFERENCES}

Abazajian K. et al., 2004, AJ, 128, 502

Ackermann M. et al., 2010, ApJ, 717, L71

Ackermann M. et al., 2016, ApJ, 819, 149

Anders E., Grevesse N., 1989, Geochim. Cosmochim. Acta, 53, 197

Arnaud K. A., 1996, in Jacoby G. H., Barnes J., eds, ASP Conf. Ser. Vol. 101, Astronomical Data Analysis Software and Systems V. Astron. Soc. Pac., San Francisco, p. 17

Blasi P., Colafrancesco S., 1999, Astropart. Phys., 12, 169

Bonafede A. et al., 2012, MNRAS, 426, 40

Bonafede A. et al., 2014, MNRAS, 444, L44 
Brienza M. et al., 2017, A\&A, 606, A98

Brunetti G., Jones T. W., 2014, Int. J. Mod. Phys. D, 23, 1430007

Brunetti G., Setti G., Feretti L., Giovannini G., 2001, MNRAS, 320, 365

Brunetti G., Blasi P., Cassano R., Gabici S., 2004, MNRAS, 350, 1174

Brunetti G., Venturi T., Dallacasa D., Cassano R., Dolag K., Giacintucci S., Setti G., 2007, ApJ, 670, L5

Brunetti G. et al., 2008, Nature, 455, 944

Brunetti G., Cassano R., Dolag K., Setti G., 2009, A\&A, 507, 661

Brunetti G., Blasi P., Reimer O., Rudnick L., Bonafede A., Brown S., 2012, MNRAS, 426, 956

Cassano R., Brunetti G., Setti G., 2006, MNRAS, 369, 1577

Cassano R., Brunetti G., Norris R. P., Röttgering H. J. A., Johnston-Hollitt M., Trasatti M., 2012, A\&A, 548, A100

Cassano R. et al., 2013, ApJ, 777, 141

Condon J. J., Cotton W. D., Greisen E. W., Yin Q. F., Perley R. A., Taylor G. B., Broderick J. J., 1998, AJ, 115, 1693

Cuciti V., Cassano R., Brunetti G., Dallacasa D., Kale R., Ettori S., Venturi T., 2015, A\&A, 580, A97

de Gasperin F. et al., 2017, preprint (arXiv:1710.06796)

Dehghan S., Johnston-Hollitt M., Franzen T. M. O., Norris R. P., Miller N. A., 2014, AJ, 148, 75

Dennison B., 1980, ApJ, 239, L93

Donnert J., Dolag K., Brunetti G., Cassano R., Bonafede A., 2010a, MNRAS, 401, 47

Donnert J., Dolag K., Cassano R., Brunetti G., 2010b, MNRAS, 407, 1565

Donnert J., Dolag K., Brunetti G., Cassano R., 2013, MNRAS, 429, 3564

Enßlin T., Pfrommer C., Miniati F., Subramanian K., 2011, A\&A, 527, A99

Feretti L., Giovannini G., Govoni F., Murgia M., 2012, A\&AR, 20, 1

Giovannini G., Feretti L., 2000, New A, 5, 335

Intema H. T., Jagannathan P., Mooley K. P., Frail D. A., 2017, A\&A, 598, A78

Jeltema T. E., Profumo S., 2011, ApJ, 728, 53

Kaiser C. R., Alexander P., 1999, MNRAS, 302, 515

Kempner J. C., Blanton E. L., Clarke T. E., Enßlin T. A., Johnston-Hollitt M., Rudnick L., 2004, in Reiprich T., Kempner J., Soker N., eds, The Riddle of Cooling Flows in Galaxies and Clusters of galaxies, preprint (astro-ph/0310263)

Keshet U., Markevitch M., Birnboim Y., Loeb A., 2010, ApJ, 719, L74

Lane W. M., Cotton W. D., Helmboldt J. F., Kassim N. E., 2012, Radio Sci., 47, RS0K04

Macario G., Venturi T., Brunetti G., Dallacasa D., Giacintucci S., Cassano R., Bardelli S., Athreya R., 2010, A\&A, 517, A43

Macario G. et al., 2013, A\&A, 551, A141

Mahony E. K. et al., 2016, MNRAS, 463, 2997

Martinez Aviles G. et al., 2016, A\&A, 595, A116
McMullin J. P., Waters B., Schiebel D., Young W., Golap K., 2007, in Shaw R. A., Hill F., Bell D. J., eds, ASP Conf. Ser. Vol. 376, Astronomical Data Analysis Software and Systems XVI. Astron. Soc. Pac., San Francisco, p. 127

Miley G., 1980, ARA\&A, 18, 165

Miniati F., Beresnyak A., 2016, IAU Focus Meeting, 29, 700

O'Dea C. P., Owen F. N., 1985, AJ, 90, 954

Offringa A. R. et al., 2014, MNRAS, 444, 606

Owen F. N., Rudnick L., Eilek J., Rau U., Bhatnagar S., Kogan L., 2014, ApJ, 794, 24

Petrosian V., 2001, ApJ, 557, 560

Pfrommer C., Enßlin T. A., 2004, A\&A, 413, 17

Planck Collaboration XXIX, 2014, A\&A, 571, A29

Pratt G. W., Croston J. H., Arnaud M., Böhringer H., 2009, A\&A, 498, 361

Rengelink R. B., Tang Y., de Bruyn A. G., Miley G. K., Bremer M. N., Roettgering H. J. A., Bremer M. A. R., 1997, A\&AS, 124, 259

Rudnick L., Lemmerman J. A., 2009, ApJ, 697, 1341

Ryu D., Kang H., Cho J., Das S., 2008, Science, 320, 909

Saripalli L., Hunstead R. W., Subrahmanyan R., Boyce E., 2005, AJ, 130 , 896

Scaife A. M. M., Heald G. H., 2012, MNRAS, 423, L30

Shimwell T. W. et al., 2016, MNRAS, 459, 277

Shimwell T. W. et al., 2017, A\&A, 598, A104

Sommer M. W., Basu K., Intema H., Pacaud F., Bonafede A., Babul A., Bertoldi F., 2017, MNRAS, 466, 996

Srivastava S., Singal A. K., 2016, preprint (arXiv:1610.07783)

Struble M. F., Rood H. J., 1999, ApJS, 125, 35

Thierbach M., Klein U., Wielebinski R., 2003, A\&A, 397, 53

van Haarlem M. P. et al., 2013, A\&A, 556, A2

van Weeren R. J. et al., 2016, ApJS, 223, 2

van Weeren R. J. et al., 2017, Nat. Astron., 1, 0005

Vazza F., Brunetti G., Gheller C., 2009, MNRAS, 395, 1333

Vazza F., Jones T. W., Brüggen M., Brunetti G., Gheller C., Porter D., Ryu D., 2017, MNRAS, 464, 210

Vikhlinin A., Markevitch M., Murray S. S., Jones C., Forman W., Van Speybroeck L., 2005, ApJ, 628, 655

Wells D. C., 1985, Nrao'S Astronomical Image Processing System (AIPS). Springer US, Boston, MA, p. 195

Williams W. L. et al., 2016, MNRAS, 460, 2385

Willingale R., Starling R. L. C., Beardmore A. P., Tanvir N. R., O’Brien P. T., 2013, MNRAS, 431, 394

Yuan Z. S., Han J. L., Wen Z. L., 2015, ApJ, 813, 77

This paper has been typeset from a $\mathrm{T}_{\mathrm{E}} \mathrm{X} / \mathrm{L} \mathrm{T}_{\mathrm{E}} \mathrm{X}$ file prepared by the author. 